\title{
La géographie de la perception dans le monde francophone: une perspective historique
}

Parmi les voies de la nouvelle géographie, la géographie de la perception est souvent assimilée aux courants de recherche d'origine britannique ou américaine. Qui n'a entendu parler le LYNCH, GOULD Ou APPLEYARD, pour ne mentionner que quelques auteurs connus? Les mêmes références apparaissent dans tous les ouvrages, négligeant les travaux publiés dans d'autres langues que l'anglais.

Certes nul ne songe à contester le rôle moteur de la «behavioral geography», mais n'oublie-t-on pas un peu rapidement certaines racines originales de cette voie de la recherche, géographie régionale, psychologie, sociologie, sémiotique, structuralisme, ethnologie et philosophie?

L'objectif principal de cet article est donc de fournir une synthèse des origines et des principales recherches en matière de géographie de la perception dans les pays francophones. Ce texte s'attache essentiellement aux principaux courants de pensée, place de la perception dans les analyses de géographie régionale, découvertes des sciences sociales et directions prises par la géographie de la perception depuis le début des années soixante-dix. Puisse cette présentation générale fournir les grandes directions des recherches et des indications bibliographiques sur un milieu souvent ignoré par les auteurs anglo-saxons par suite des barrières linguistiques ${ }^{1)}$.

\section{Géographie régionale et interprétation du vécu}

N'affirme-t-on pas souvent que les pays à tradition cartésienne ont été longs à se mettre à l'analyse des relations subjectives que l'homme entretien avec le milieu? N'oublie-t-on pas alors rapidement que Descartes lui-même avait constaté la subjectivité de la perception et la nécessité d'une distinction entre perception et jugement?

Nul ne doit alors s'étonner à la lecture de géographes du début du siècle, comme v. CORNETZ (1913) et E. RABAUD (1927) de l'intérêt manifesté pour les phénomènes de perception et plus précisement d'orientation, d'identification et de différenciation. Toute une géographie du sens des lieux est déjà née; elle n'est pourtant pas dominante dans les décennies suivantes, écrasée

1) Nous éviterons, par contre, de faire référence aux travaux anglosaxons fort connus des lecteurs. par la géographie régionale et ses analyses volontairement empiriques et exhaustives. Néanmoins n'oublions pas que la perception n'est pas absente dans les travaux de la géographie régionale: toutes les thèses présentées soit sur les régions françaises, soit sur les pays des anciennes colonies, évoquent ce thème: étude des coïncidences entre aires physiques et culturelles (P. FONCIN, 1898), correspondances entre régions naturelles et noms de pays (L. GALLOIS, 1908). Même si la primauté est donnée aux phénomènes physiques, les auteurs s'attachent aux origines de la toponymie, aux aires d'extensions culturelles. Place est faite aux interprétations vécues, perçues à travers l'histoire, les témoignages de la vie collective, pour illustrer l'origine des divisions géographiques, la trace de leurs racines dans le milieu.

Dès 1925 , dans sa morphologie du paysage, C. SAUER donnait comme champ d'étude à la géographie, l'organisation systématique du contenu du paysage et de ses causes. Mais cette géographie causale n'était pas, comme certains l'ont interprété, une véritable science positive. Même si la méthode morphologique devait opérer dans de strictes limites naturalistes, son paysage n'excluait pas l'imagination, les images introspectives d'un monde subjectif. Deux orientations philosophiques majeures marquent alors l'analyse du paysage, positivisme et phénoménologie. Peut-on se contenter de l'étude des objets tels qu'ils sont ou doit-on chercher à comprendre dans les forces qui ne sont pas directement observables, celles qui sont clairement subjectives?

Il s'agit en général, en géographie régional, d'études consacrées à de petites sociétés, à travers les sentiments d'appartenance régionale, la psychologie collective, l'analyse des attitudes et des institutions (DEMANGEON, 1923), sans qu'elles puissent pourtant se détacher du naturalisme et de la croyance utopique en une pseudo-objectivité du chercheur. En aucun cas la psychologie individuelle n'est privilegiée, par crainte de promouvoir les croyances personnelles et marginales. Le chercheur lui-même n'est-il pas considéré comme un observateur impartial, garant de la neutralité scientifique...!

L'unanimité des successeurs de DE MARTONNE et de V. DE LA BLACHE n'est pourtant pas totale. Des auteurs

Antoine S. Bailly, professeur à l'Université de Genève, 15, rue Butini, 1202 Genève. 
comme J. BRUNHES n'insistent-ils pas sur les croyances locales et même le folklore pour en saisir toute la subjectivité? DEFFONTAINES (1968) ne s'interroge-t-il pas sur les attitudes des civilisations face à la vie et face à la mort? Toute une géographie de la spiritualité, composante de la géographie culturelle, est ainsi implicite dans une partie de l'approche régionale, comme l'illustrent également les travaux de J. GALLAIS (1968) sur l'espace vécu du peuple Peul dans les régions soudano-sahéliennes. C'est par une connaissance profonde de la langue peule que l'auteur arrive à s'attacher à l'organisation des unités territoriales fondamentales, les leydus. J. GALLAIS pose alors un problème essentiel, souvent ignoré en géographie régionale et même en géographie active: comment travailler sur son propre milieu, sinon à travers sa propre subjectivité et avec tous les risques de biais qui en résultent? Ne vaut-il pas mieux s'attacher à la subjectivité des autres, en décryptant leurs tissus de relations? A chaque culture ses perceptions, ses valeurs et même ses échelles géographiques qui permettent à J. GALLAIS de différencier au Sahel la notion de distance affective (selon la fragmentation des ethnies) de celle de distance écologique, reposant sur les nuances du milieu.

Quelques auteurs de la géographie régionale française ont donc implicitement fait une place à la perception, en cherchant à découvrir ce qui attache l'homme à la terre. Ils ouvrent ainsi, un peu malgré eux, la voie aux analyses de la subjectivité individuelle et sociale et à une nouvelle direction de recherche, incorporant les découvertes de la psychologie de la perception, de la phénoménologie et de la sémiotique. Voyons donc tout d'abord les principaux courants de pensée des sciences sociales connexes, avant de nous attacher à leurs influences sur la géographie contemporaine.

\section{Les sciences sociales à la recherche des processus cognitifs}

\subsection{Les découvertes de l'analyse psychologique}

Il n'est pas besoin de rappeler en détail le rôle moteur des travaux de PIAGET (PIAGET, 1936; PIAGET et INHELDER, 1948) sur la perception de l'espace chez l'enfant et leur construction progressive des bases de la géométrie pour illustrer leur influence sur la pensée contemporaine dans les sciences sociales. Ces recherches sur la genèse et la structure des images spatiales ont donné naissance à de multiples expérimentations sur la formation des enfants, afin de saisir le rôle de l'apprentissage, des filtres sociaux, pour expliquer leurs attitudes au foyer, à l'école. Est ainsi posée la question de l'acquis, par opposition à l'inné (SKINNER) et indirectement celui du choix entre des actions possibles. Parmi les travaux moins connus hors des pays francophones et datant de la même époque que ceux de l'école de Genève, mentionnons ceux de P. Guillaume (1937) sur la psychologie de la forme (Gestalt) et ceux de R. FRANCĖs (1963) sur l'organisation perceptive, son aspect évolutif, la signification des objets, le rôle des motivations et l'autonomie de la perception sociale. La puissance de ces courants de réflexion a été l'entrave majeure au développement de la théorie mécaniste Stimulus-Réponse dans les pays francophones; l'approche fait appel à la théorie de l'information pour saisir les mécanismes d'apprentissage, de décodage des signaux en bruits, pour intégrer le temps et les processus de rétroaction et pour poser les problèmes de perception en termes sociaux et culturels.

Un exemple frappant est donné par l'ouvrage de PaILHOUS (1970), véritable psychologie du travail en milieu urbain. Par l'étude des cheminements dans la ville des chauffeurs de taxis anciens et débutants, de la structure de leurs images spatiales et du rôle fonctionnel de leurs chaînes d'opérations, J. PAILHous constate le rôle d'un réseau routier principal (ou réseau de base), trame recouvrant la ville. A mesure de l'approfondissement de son expérience, ce réseau de base se précise, avec pour corollaire la réduction du réseau secondaire (pénétrantes à l'intérieur des quartiers) qui ne sert plus qu'à rejoindre le réseau de base. Le sujet opère par lecture de coordonnées à partir des repères (carrefours) et d'alignements de points, qui permettent d'établir des relations topologiques de voisinage entre les aires (quartiers). A chaque carrefour le chauffeur choisit ainsi la voie principale qui fait l'angle minimum avec l'estimation du but (règle algorithmique). Ainsi le citadin reconstruit-il la ville en fonction de sa pratique des déplacements en codant les informations du plan. L'image constitue alors le support essentiel des opérations du sujet.

Des études à propos de l'adaptation de l'homme à l'espace sont également développées par des auteurs comme LECUYER (1975) dans un article très critique des travaụx de LORENZ. Les analogies trop rapides du comportement humain à celui des animaux font oublier l'importance des relations et des informations sociospatiales. Au lieu d'espace personnel (MOLES et ROHMER, 1972), l'auteur préfère parler de distance interpersonnelle. Ce que l'on observe dans les relations humaines ce sont des distances interpersonnelles compatibles avec certains modes de communication. Remise en cause des travaux de E. HALL, la préoccupation de LECUYER est de comprendre les relations humaines à travers les dynamiques sociales.

On trouve un objectif semblable, à une autre échelle géographique, dans l'ouvrage de MOLES et ROHMER (1976) sur la micropsychologie de la vie quotidienne. La décomposition des grandes actions de notre vie sociale en un flux presque ininterrompu de démarches mineures, de feuilles à remplir, de guichets à fréquenter, dilue la grandeur au niveau du train-train quotidien. L'analyse de l'attente, acte quotidien, illustre toute une série de microcomportements humains, sans lesquels on ne peut saisir les réactions de violence, d'agressivité. Il serait impossible de citer ici toutes les études des multiples relations et agressions imposées aux hommes par la société contemporaine; mention- 
nons simplement par exemple la perte d'identité décelée par LEROY et SIVADON des personnes hospitalisées longtemps et coupées du milieu extérieur, les effets du bruit sur les retards scolaires près de l'aéroport d'Orly, les problèmes pathologiques liés aux changements rapides de crèches et de gardiens chez les petits enfants (nanisme affectif) et les troubles qu'éprouvent les vieillards en cas de déplacement spatial forcé. A chaque niveau, dans chaque exemple une constante, l'insertion obligatoire de l'homme dans un espace social qui limite sa liberté intersticielle. Corps et territoires sont intimement liés; on est ainsi d'abord de sa famille, de son groupe socio-culturel, de sa ville, de sa région, de son pays... Chaque changement de territoire et de ses contraintes est alors susceptible d'engendrer des pathologies dont les cas les plus connus sont par exemple liés aux migrations.

\subsection{Sociologie, sémiotique et structuralisme}

Les sociologues se sont naturellement intéressés, quoique après les psychologues, aux relations hommeenvironnement dans un contexte social et plus particulièrement à la signification des images et à l'analyse des signes de la ville. Outre CHOMBART DE LAUWE (1963), pionnier dans les enquêtes de quartiers, citons LEDRUT et la ville rêvée des toulousains (1973). L'analyse sémantique des signes de la ville nous apprend d'ailleurs plus sur les citadins, sur leur vécu individuel et collectif, sur le symbolisme des actes, que sur la ville elle-même. Le centre est devenu une vitrine à valeur marchande dans laquelle chacun, suivant son sexe, son milieu, son mode de vie, son statut socio-économique, choisit ses lieux préférés.

L'attachement des citadins à la résidence et leurs relations au centre-ville deviennent alors les thèmes majeurs de la sociologie urbaine des pays francophones. DEBRUELLE et ROGGEMANS (1974) mènent par exemple une enquête, en mai 1970 à partir d'un échantillon représentatif de 2269 personnes, pour définir les besoins et les aspirations des Bruxellois. Les types distincts d'intégration sont le fait de groupes et sousgroupes sociaux bien définis, jeunes et personnes issues de milieux favorisés particulièrement attirés par le centre, ouvriers et citadins âgés qui le sont moins.

Quatre classes sont dégagées correspondant à trois variables principales, âge, catégorie socio-professionnelle, stabilité résidentielle:

- $47 \%$ des personnes enquêtées sont attachées au lieu de résidence (plus petit que le quartier), mais moins au centre-ville;

- $22 \%$ des personnes enquêtées sont non-intégrées au quartier et au centre-ville;

- 19\% des personnes enquêtées ont un comportement traduisant une polarité complémentaire entre le lieu de résidence et le centre-ville;

- $12 \%$ des personnes enquêtées sont peu attachées au quartier de résidence, mais plus au centre-ville.
Place est faite, dans toutes ces études, à la démarche structuraliste dans l'espoir de trouver un nouveau modèle applicable à la compréhension des images de la ville et des systèmes de représentation-communication. Ces recherches se situent d'ailleurs aux confins de la sociologie, de l'urbanisme et de la linguistique. La sémiotique en particulier a brisé ses attaches directes avec la linguistique pour se consacrer à tous les processus de communication. L'évolution des modes de représentation présentée par J. ZEITOUN en 1973 lors d'un colloque sur la Sémiotique de l'espace (1979) est par exemple destinée à préciser le statut et la structure des objets topologiques selon les deux dimensions correlées du signifiant spatial et du signifié culturel. Ce rapport du signifiant et du signifié est central dans les recherches à propos de l'espace de C. Soucy (1967 et 1971) qui se réfère aux méditations de BARTHES sur les mythologies de la ville langage (1957) et sur les significations comparées du centre dans les villes occidentales et au Japon (1970). A travers quatre auteurs (BUTOR, LE CLEZIO, C. ROCHEFORT et PEREC), C. SOUCY dégage les éléments pour une analyse structurale du discours: le centre urbain est perçu comme le lieu idéal de consommation de signes, le lieu de projection des discours des groupes dominants. L'exploitation de ce centre, traduite par le choix de symboles, résulte alors des stratégies de groupes. Ainsi comprend-t-on l'image que les promoteurs immobiliers cherchent à imposer à leur clientèle potentielle à travers la publicité immobilière: quartiers bien habités, calmes (même à proximité du centre), cadre de vie plaisant, panorama sur des zones préservées de nature, de loisirs...

Il n'y a plus de discours de la ville, mais des discours sur la ville émanant des divers groupes qui la fréquentent et parmi eux surtout les plus favorisés qui disposent des moyens de médiation culturelle. Peut-être cela semble-t-il bien classique dans la littérature actuelle, mais n'oublions pas que l'éclosion de ce type de discours date des années cinquante. Le paysage urbain (L'Espace Géographique, 1974) est depuis cette époque conçu essentiellement comme code de signes adaptés à certains membres de la société; la création de ces signes, donc des symboles, échappe à la majorité des individus pour se concentrer dans des lieux où agissent les groupes dominants; pour les autres personnes issues de classes sociales, économiques, ethniques différentes, les groupes dominants imposent des codes extérieurs (verbaux, graphiques) pour rendre le milieu lisible et «consommable». L'espace se charge alors de valeurs économiques privilégiées par la société productiviste, mais incapables de répondre aux valeurs profondes des individus.

La sémiologie spatiale renvoie ainsi aux groupes qui se partagent l'espace et aux moyens mis en œuvre (puissance) pour assurer le pouvoir. Ainsi s'esquisse une science de l'information, du message, attachée à l'étude de la structure d'une société différenciée aux objectifs hétérogènes. Le paysage devient l'ensemble des signes dans lesquels il convient de recher- 
cher les signifiés. Ce paysage est cependant bien incomplet (BRUNET, 1974, in l'Espace Géographique) car tous les signifiés (structure sociale) ne se traduisent pas par des signifiants. A. LÉVY (1978) explique la complexité du phénomène par une double articulation qui comprend deux plans et deux axes:

- un plan de l'expression, le signifiant spatial urbain;

- un plan du contenu, la signification socio-sémiotique;

- un axe de système qui consiste dans les catégories paradigmatiques (urbanistiques par exemple);

- un axe du procès qui consiste dans les relations syntagmatiques (cumul de propriétés distinctives).

\subsection{Ethnologues et philosophes à la recherche du sens de l'espace}

Bien des ethnologues (BALZET et al., 1976) croient également au rôle majeur des relations dissymétriques pour s'attacher par exemple comme BougHALI (1974) aux représentations de l'espace dans les communautés de pays du tiers-monde et du bassin méditerranéen. A l'aide d'une matrice comparative incorporant des variables sur l'espace donné (forme, proportions de l'habitat, équilibre spatial des ouvertes, agencement des pièces...) et sur l'espace produit (ordonnance symbolique du milieu, sociabilité, représentation...) et permettant de mettre en comparaison les Cévennes, la Provence et la Corse, H. BALZet (1976) s'attache au processus de transformation économique, sociologique, cognitif des communautés méditerranéennes. Sentiment d'appartenance, cercles de référence, poids de l'insertion locale contrastent avec les zones éloignées psychologiquement, considérées comme étrangères. Toute une typologie des «lointains» peut ainsi être établie à partir des convergences et variations socioculturelles.

C'est cette nécessité de plonger dans les racines du passé, pour éviter la banalisation, la désacralisation de la vie moderne, qui pousse M. BOUGHALI (1974) à étudier la représentation de l'espace chez le marocain illettré. Toute une dimension spatiale est présente dans les rites traditionnels, tant lors de l'accouchement dans un grand plat de terre couvert d'un tissu blanc, qu'au moment du rejet de cette terre hors de la maison pour se protéger et annoncer aux forces invisibles cette nouvelle arrivée. Le caractère sacré de l'espace et du temps, facettes complémentaires d'une même existence, rythme la vie de la naissance à la mort. C'est en reconstituant les racines profondes de la tradition que l'on comprend les sources psycho-sociologiques des comportements territoriaux et que l'on évite la destruction des systèmes culturels traditionnels. Un lieu, quel qu'il soit, n'est rien pris en lui-même. Il est porteur de sens par ses relations avec d'autres lieux, d'autres hommes. Les axes de lecture du monde sont sélectionnés à travers notre culture (imaginaire) qui renvoie aux pratiques sociales et à l'histoire. Ainsi peut-on à la manière de BACHELARD (1957) songer à une poétique de l'espace, cadre de vie vivant où chaque portion prend une valeur signifiante. Nul discours à prétention scientifique dans ce cas, comme dans celui de SANSOT (1973), l'espace parle librement à travers la description. La méthode est inspirée de l'esthétique descriptive, marquée par les dualités entre observantobservé et signifiant-signifié. La poétique est une qualité de certains lieux qui introduit l'homme, son travail, ses joies, ses peines dans les typologies au lieu de chercher l'objectivité réductrice de la démarche scientifique qui détermine arbitrairement son champ d'investigation. Prostituées, clochards, autant de sujets qui permettent d'esquisser une tentative de libération dans un monde d'aliénation, des échappatoires au désespoir.

La symbolique de la circulation réintroduit l'épaisseur poétique du faubourg, de la rue, celle de la résidence secondaire (SANSOT et. al., 1978), une forme caricaturée et rigidifiée du principe d'habiter. L'espace secondaire, comme le loisir sont d'abord des productions de l'imaginaire, inhérentes à toute vie sociale. Par l'appropriation de l'espace, «le champ secondaire privilégie une consommation de biens ou de signes qui ne sont pas du domaine principal». L'ingéniosité des classes au pouvoir est de faire acquérir au secondaire une légitimité par la mise en «espace-frontière» d'un phénomène diffus. La logique du fonctionnel, qui guide notre société contemporaine, s'étend bien audelà du travail et de la production, dans l'espace et dans la durée de notre vie quotidienne. Reste-t-il encore en nous place pour un espace et un temps intime? La nuit peut-être... (CAUQUELIN, 1977).

A travers la variété des recherches des sciences sociales, il peut apparaitre des faiblesses de corpus théorique, des incertitudes méthodologiques, et même un manque d'homogénéité dans les résultats. Pourtant s'arrêter à ces conclusions serait superficiel, voire erroné. Partout les optiques structuralistes et celles des sciences sociales critiques sont privilégiées. Plus réticents que leurs confrères anglo-saxons à accepter les perspectives mécanistes de l'environnementalisme et les idéologies individualistes, les chercheurs francophones insistent avant tout sur le rôle de la société et la faiblesse de la liberté interstitielle qui en résulte. Est-ce lié à une longue tradition marxiste ou à un type de recherche privilégiant les analyses de groupes plutôt que celles des individus? Sans doute aux deux puisque dans chaque exemple la perspective sociale est centrale dans les hypothèses et les interprétations.

\section{La géographie de la perception dans les pays francophones}

La géographie francophone ne pouvait longtemps ignorer de tels courants de pensée même si elle optait pour la logique inductive des analyses régionales. Sans doute plus tardivement que dans les pays anglo-saxons, 
mais avec des perspectives différentes, elle s'est lancée dans la recherche des phénomènes de perception. Il n'est pas surprenant de constater que les principaux travaux soient consacrés à la territorialité régionale et urbaine, thèmes qui intéressaient déjà les géographes. Deux types de méthodes dominent, celle de la psychologie pour saisir le vécu individuel des hommes et celle, plus poétique, de l'interpretation à travers des textes des sentiments exprimés par des auteurs. Voyons tout d'abord ce deuxième cas, avant de nous consacrer aux recherches plus expérimentales du premier cas.

\subsection{Les géographies de l'imaginaire}

C'est A. FRÉmONT qui développe le mieux dans «La région espace vécu» (1976) l'introduction de l'affectivité, du symbolisme dans une géographie régionale trop longtemps marquée par le positivisme logique et la place du milieu naturel. Ici la rupture avec la géographie physique est consommée, la perspective centrée sur les acteurs se situe délibérément dans le courant culturel des sciences sociales qui renvoie aux pratiques sociales et à l'histoire. Le sens des lieux dépend des relations tissées entre hommes et milieux; ainsi l'auteur distingue-t-il les régions fluides - sans ancrage des hommes aux lieux -, les régions enracinées - les lieux appartiennent aux hommes et les hommes aux lieux -, et les régions fonctionnelles - espaces de la société industrielle -. L'interprétation repose sur de nombreuses études de cas menées tant au plan régional (Séminaire de Recherche de BÉNOUVILLE, 1973), qu'à travers les textes littéraires (FRÉMONT, 1980). Par exemple les images de la Normandie varient suivant les acteurs, administrateurs, créateurs d'emploi, grand public, parisiens. Côté positif on note le calme, la tranquillité, la campagne, la gastronomie, la présence de la mer, l'habitat individuel, la douceur de vivre, côté négatif l'ennui, le conservatisme moral, la passivité des habitants, l'absence d'industries et d'équipements, le climat humide... La liste pourrait être plus longue, mais ce qui importe c'est le point de vue choisi par l'acteur. Ainsi MAUPASSANT (FRÉMONT, 1980) traduit-il la plénitude d'une société paysanne, aux valeurs établies, qui permettent à la fois de régler l'ensemble des rapports sociaux, les relations entre paysans, notables, citadins, et le rôle de l'argent et du plaisir.

Dans cette géographie du vécu territorial, les références littéraires sont nombreuses, Madame BOVARY à Rouen pour A. FRÉMONT, les romanciers et les poètes du XIXe et $\mathrm{XXe}$ siècles et les romans policiers pour A. BAILLY (1977), et même la bible pour J.-L. PIVETEAU (1978). La lecture est utilisée comme un témoignage surtout lorsqu'un espace précis est objet d'écriture. J.-L. PIVETEAU montre alors comment, dans la seconde moitié du VIe siècle, l'espace vécu du peuple hébreu combine la trace d'une sédentarisation graduelle, des traits d'une conjoncture politique mouvementée, des représentations des relations avec Dieu qui permettent l'emprise concrète sur un sol défini. La rentrée en possession de cet espace provient de l'élection, de la grâce divine, l'exil étant à l'opposé le signe de la disgrâce.

Dans chacune des formes littéraires analysées, A. BAILLY (1977) dégage des préoccupations, des thèmes communs aux auteurs. Apparaît ainsi le souci de restituer l'échelle géographique (par la vision à vol d'oiseau ou la déambulation), de disposer des repères symboliques et personnels, et de présenter des schémas logiques de fonctionnement. La perception de l'espace se fait essentiellement à travers les contrastes soulignés par l'auteur: la région, la ville, miroirs de la société seront à la fois rêve et cauchemar.

J.-M. HOLZ (1980) retrouve cette spatialité, aux cellules multiples, aux champs de forces avec ses productions, ses discountinuités dans son voyage dans l'espace. L'espace vécu est alors également espace aliéné, parfois même il perd sa signification propre. R. FERRAS (1977) explique alors comment l'image de la garrigue des enfants du midi de la France reflète plus l'image des manuels français imposés dans l'éducation - nature passe-partout, campagne idyllique - que celle de la réalité méditerranéenne.

Toute l'influence de la symbolique de nos sociétés est présentée également dans les publicités touristiques. L'espace touristique est avant tout image: image globale, dialectique subtile entre la ville et la nature, l'ordre et le désordre, le traditionnel et le moderne (MIOSSEC, 1977). G. CAZES (1974) découvre dans les publicités touristiques à propos de pays du tiers-monde, une conception insistant à la fois sur la culture du client potentiel et une caricature de l'exotisme qui occulte les réalités socio-politiques. Des mythologies de même type sont d'ailleurs développées pour des aires très différentes, par exemple la montagne étudiée par J.-P. GUÉRIN et H. GUMUCHIAN (1977 et 1978), à partir d'un dépouillement informatique intitulé «approximation automatique de l'analyse du discours». La publicité hivernale s'articule autour du soleil, du ski, de la station alors que l'été, elle est plus culturelle, intégrant certains éléments de la vie locale, des paysages. La montagne devient le conservatoire de la tradition, de l'écologie, les véritables problèmes économiques n'étant jamais mentionnés. Les estivants, comme le personnel acceptent d'ailleurs ce discours ritualisé: le séjour du vacancier est une croisière de ski, soleil, sommeil, tout comme celui des employés qui se sentent, pour un moment de leur vie professionnelle, dans un lieu privilégié dont ils se considèrent connaisseurs et maîtres.

Par l'étude de l'imaginaire, de l'espace vécu, le géographe aborde ainsi les composantes sociales de l'image, les rôles des groupes dominants dans les relations sociales et la forme dialectique des rapports hommemilieu. Les auteurs, nous l'avons déjà vu pour GUÉRIN et GUMUCHIAN (1977) font alors appel aux méthodes de la sémiologie pour dresser de véritables typologies des images perçues. 


\subsection{Les géographies de la territorialité urbaine}

Quartiers et centres urbains ont été les sujets du plus grand nombre d'études, utilisant les méthodes de la psychologie, tentatives de typologies spatiales, rechercher les motivations et les stratégies commerciales. BERTRAND et METTON (1974), en s'aidant de repères, ilustrent cette première tendance en plaçant sur un plan les limites de chaque représentation individuelle. A l'aide de l'axe le plus long (AP) et de son milieu (M), de l'axe secondaire (BS) et du lieu de domicile (D), trois indices sont calculés:

$\begin{aligned} \text { superficie } & =\mathrm{AP} \times \mathrm{BS} \\ \text { forme } & =\frac{\mathrm{BS}}{\mathrm{AP}} \\ \text { centration } & =\frac{\mathrm{DM}}{\mathrm{AM}}\end{aligned}$

Ces indices se prêtent bien aux comparaisons de la surface des quartiers vécus: plus le quartier est circulaire, plus l'indice tend vers 1 , plus le domicile est centré, plus l'indice tend vers 0 .

Cet indice constitue un moyen pour mieux saisir les pratiques de la ville (BERTRAND, 1978). Ainsi les grands ensembles sont-ils perçus par leurs habitants de manière circulaire à partir d'un paysage ouvert de places, de pelouses, de stationnements. L'aire vécue se limite aux abords visibles de l'habitation et se réduit aux nuisances, les équipements étant mal connus, ce qui explique la faiblesse des choix individuels.

R. FERRAS et M. VIGOUROUX (1978) tentent, avec d'autres méthodes graphiques, de comprendre, à propos de deux villes du Languedoc, Béziers et Sète, le processus de formation des cartes mentales. Il se compose de trois éléments majeurs:

- l'environnement et ses composantes vécues par l'individu;

- l'idéologie;

- les caractéristiques individuelles (statut social, âge...).

A partir d'un échantillon de retraités et d'enfants choisis en milieu urbain, R. FERRAS constate la communauté des structures d'ensemble. La perception du centre passe tout d'abord par des lieux bien repérés et bien désignés (place de la Comédie où l'agora se perpétue) et par des consommations fonctionnalistes qualifiées par l'appareil commercial. Seul le paysage mineur diffère par suite d'intérêts immédiats, essentiels par exemple lors d'un cheminement. Ces images communes doivent beaucoup aux modèles et principes culturels (stéréotypes de l'imaginaire social). Cette homogénéisation est d'autant plus grande que le rôle des promoteurs commerciaux accélère le processus de concentration commerciale au centre, tendant à interdire un rôle actif dans le centre aux populations défavorisées. Ainsi note-t-on à Mulhouse (BAILLY et WOESSNER, 1979) deux images du centre correspondant à deux classes de statut socio-économique aux fréquen- tations différentes. X. PIOLLE (1977) illustre à propos de Pau le renforcement de ces ségrégations, les populations aux revenus modestes réduisant à peu de choses leur espace central.

Il devient alors essentiel de savoir qui privilégie certaines fonctions commerciales du centre et certaines fonctions secondaires d'animation-culture-loisirs pour déboucher sur les motivations des citadins. Quatre groupes de variables expliquent pour MERENNESCHOUMAKER, (1974 et 1979) les images différentes:

- l'âge, car les enfants et les personnes âgées ne perçoivent pas la même ville que les adultes;

- le sexe, les femmes attachant de l'importance à la fonction commerciale, les hommes aux affaires et aux loisirs;

- le niveau socio-professionnel, par suite d'une image intellectualisée pour les professions libérales, et liée au vécu quotidien pour les ouvriers;

- les caractères socio-géographiques, la durée du séjour élargissant les limites du centre, l'origine rurale entraînant une image plus positive du centre que celle des ouvriers.

Les commerçants, conscients de ces différences, se localisent alors en fonction de la centralité, de la qualité de l'environnement commercial et dans une moindre mesure non commercial et de l'intensité des flux de fréquentation. Il n'est donc pas étonnant de constater l'apparition de hiérarchies de nodules commerciaux, avec à la base le trio boulangerie-boucherie-épicerie, qui permet presque n'importe où en Europe Occidentale de recréer un espace vécu à partir d'associations commerciales semblables (METTON, 1977).

$\mathrm{Au}$ delà des images à composantes fonctionnelles, liées au statut social, BELANGER et GENDREAU (1978) montrent que les résidents, comme les touristes, outre le souhait de bénéficier d'un spectacle architectural et d'un choix commercial, sont également sensibles à la qualité de la communication humaine. L'ambiance, c'est-à-dire le rôle des gens dans le paysage, les liens entre un peuplement et un territoire sont aussi importants que les éléments architecturaux. De là le rôle de la déambulation, de la promenade détendue dans le centre, de là également le souci de globalité pour tout aménagement urbain. La géographie de la perception débouche alors sur une étape active proposant des recommandations. C'est ce que font BÉLANGER et GENDREAU (1978) pour la ville de Québec, donner une priorité aux transports collectifs et créer des rues piétonnières, et BAILLY (1977) pour Belfort, en découpant la ville en secteurs homogènes en vue d'un plan d'aménagement urbain et en dressant des listes des éléments fondamentaux à prendre en compte dans l'aménagement de ces secteurs (rénovation, animation, créations d'espaces verts, d'aires commerciales et de services...). Ces méthodes permettent dans la plupart des études d'aménagement d'enrichir la consultation du public et de prendre en compte 
l'image des individus au lieu de constater ex-post la résignation d'habitants opposés à certains changements urbains (PAQUETTE et al., 1974).

Ces quelques exemples illustrent le souhait des géographes francophones, non seulement de comprendre, à travers des approches structuralistes, comment le tissus urbain est perçu, mais surtout d'intégrer leurs réflexions dans le cadre des relations sociétales pour déboucher sur des recommandations concernant l'aménagement de l'espace. La géographie de la perception qui s'attache à la fois aux rôles des hommes et à leurs places dans la société fait partie des sciences sociales critiques. D'autres exemples auraient pu illustrer la variété des travaux menés dans les pays francophones - perception des milieux ruraux, des paysages naturels... -, mais dans un texte synthétique des choix sont à faire et seuls les courants de pensée qui nous semblaient les plus importants ont été traités. Souhaitons qu'ils continuent à être développés, parallèlement aux autres voies de la nouvelle géographie, pour conserver à l'homme sa place majeure dans le courant des sciences sociales.

\section{Résumé}

La géographie de la perception dans le monde francophone: une perspective historique.

L'objectif principal de cet article est de fournir une présentation synthètique des origines et des principales voies de recherche en géographie de la perception dans les pays francophones. Place de la perception dans la géographie régionale, découvertes des sciences sociales (psychologie, sociologie, sémiotique, structuralisme, ethnologie et philosophie) et directions prises par la nouvelle géographie «behavioriste» sont successivement analysées.

\section{Abstract}

Behavioral geography in the French speaking world: a historical approach.

This paper deals with the origins and the main directions of research in behavioral geography in the French speaking world. Behavioral trends in regional geography, social sciences (such as psychology, semiology, sociology, ethnology and philosophy) and in new geography are analyzed in this historical approach.

\section{Bibliographie}

\section{Géographie régionale}

CORNETZ, v. (1913): Le cas élémentaire du sens de la direction chez l'homme, Bulletin de la Société de Géographie d'Alger, 18e année.

DEFFONTAINE et al.(1968): Géographiegénérale, N. R.F., Collection La Pléiade, Paris.
DEMANGEON, A. (1923): L'empire britannique: étude de géographie coloniale, A. Colin, Paris.

ESPACE GÉOGRAPHIQUE (1974): numéro spécial sur la Géographie de la perception, no 3, p. 179 à 240 (articles de P. CLAVAL, R. BRUNET, A. BAILLY, A. METTON).

FONCIN, P. (1898): Les pays de la France, A. Colin, Paris. Gallais, J. (1968): La delta intérieur du Niger. Étude de géographie régionale, Mémoires de L'IFAN, Dakar. GALLOIS, L. (1908): Régions naturelles et noms de pays, A. Colin, Paris.

RABAUD, E. (1927): L'orientation lointaine et la reconnaissance des lieux, Masson, Paris.

SAUER, C. (1925): The morphology of landscape in Leighley J. (ED.), 1963, Land and life, University of California Press, Berkeley.

\section{Sciences sociales}

BACHELARD, G. (1957): La poétique de l'espace, P. U.F., Paris.

BALFET, H. et al. (1976): Pratiques et représentations de l'espace dans les communautés méditerranéennes, Ed. du C.N.R.S., Paris.

BARTHES, R. (1957): Mythologies, Seuil, Paris.

BARTheS, R. (1970): L'empire des signes, Skira, Paris. BoUghaLI, M. (1974): La représentation de l'espace chez le marocain illettré, Anthropos, Paris.

CAUQuelin, A. (1977): La ville, la nuit, P. U.F., Coll. La Politique éclatée, Paris.

CHOMBaRT DE LAUWE, P.H. (1963): Des hommes et des villes, Payot, no 154, Petite Bibliothèque, Paris.

DELRUELLE, N. et ROGGEMANS M. (1974): L'agglomération bruxelloise: approche géographique et sociologique, Ed. de l'Université de Bruxelles, Coll. du Centre de Sociologie, Bruxelles.

ESPACE GÉographiQue (1974): Numéro Spécial, no 2, Paysages et sémiologie, p. 113-151.

FRANCES, R. (1963): La perception, P. U. F., Que Sais-je?, Paris.

Guillaume, P. (1937): La psychologie de la forme, Flammarion, Paris.

LECUYER, R. (1975): Psychosociologie de l'espace, adaptation de l'espace à l'homme, Annales de Psychologie, 75, p. 549-573.

LEDRUT, R. (1973): Les images de la ville, Anthropos, Paris.

LEVY, A. (1978): Introduction à une socio-sémiotique de la perception de l'espace urbain, Espaces et Sociétés, no 24 , p. $125-130$.

LEVY-LEBOYER, C. (1980): Psychologie et environnement, P. U. F., Le Psychologue, Paris.

MAFFELOSI, et al. (1979): Espaces et imaginaire, Presses Universitaires de Grenoble, Grenoble.

MOLES, A.et ROHMER, E. (1972): Psychologie de l'espace, Castermann, Tournai. 
MOLES, A.et ROHMER, E. (1976): Micropsychologie et vie quotidienne, Denoël Gonthier, Paris.

PAILHOUS, J. (1970): La représentation de l'espace urbain: l'exemple du chauffeur de taxi, P. U. F., Paris.

PIAGET, J. (1936): L'origine de l'intelligence chez les enfants, Delachaux et Nestlé, Neuchâtel.

PIAGET, J. et INHELDER B. (1948): La représentation de l'espace chez l'enfant, P. U. F., Paris.

PRIETO, L. (1975): Messages et signaux, P.U.F., Paris. RAFFESTIN, C. (1978): Notions, concepts et construits en géographie humaine, Géopoint, publié au Centre Universitaire d'Avignon, p. 55-74.

SANSOT, P. (1973): Poétique de la ville, Klincksiek, Paris. SANSOT,P.,STROHL,H.,TORGUE, H.et VERDILLON,C. (1978): L'espace et son double, Ed. du Champ Urbain, Paris. SCHAFF, A. (1971 et 1972): La traduction et le sens de la VIe thèse sur Feuerbach, L'homme et la société, no 19 à no 24/25 (avec réponse de L. Sève à A. Schaff). SĖVE, L. (1972): Marxisme et théorie de la personnalité, Editions Sociales, Paris.

SOUCY, C. (1967): Un art de vivre unique au monde, Communications, Vol. 10, p. 135-154.

SOUCY, C. (1971): L'image du centre dans quatre romans contemporains, C. S. U., Paris.

ZeItoun, J. et al. (1979): Sémiotique de l'espace, Denoël Gonthier, Paris.

\section{Géographie de la perception}

BAILLY, A. (1977): La perception de l'espace urbain: les concepts, les méthodes d'étude, leur utilisation dans la recherche géographique, C. R. U., Paris, (texte intégral publié en 1980, Université de Lille III).

BAILLY, A. (1979): La perception des transports en commun par l'usager, T. E. C., no 32, p. 23-29.

BAILLY, A., RAFFESTIN, CL., REYMOND, H. (1980): Les concepts du paysage: problématique et représentations, L'Espace Géographique, no 4, p. 277-286.

BAILLY, A.et WOESSNER, R. (1979): Images du centreville et méthodes d'analyse factorielle: le cas de Mulhouse, Environment and Planning A, p. 1039-1048. BELANGER, M. et GENDREAU, A. (1978): Le réaménagement du vieux-Québec et l'analyse de la perception de l'environnement, L'Espace Géographique, no 2, p. 85-92.

Bertrand, M. (1978): Pratique de la ville, Masson, Paris.
CAZES, G. (1974): Le tiers-monde vu par les publicités touristiques: une image géographique mystifiante, Travaux de l'Institut de Géographie de Reims, no 20, p. 5-46.

FERRAS, R. (1977): L'enfant et la garrigue: à propos de l'espace vécu, Colloque de Rouen.

FERRAS, R. (1978): Ecusson et polygone, Bulletin de la Société Languedocienne de Géographie, Tome, XII, fasc. $1 / 2$.

FREMONT, A. (1976): La région espace vécu, P.U.F., Paris.

FREMONT, A. (1980): Le pays de Caux de Maupassant: des lieux et des hommes, Etudes offertes à Louis Papy. GUERIN, J.-P. et GUMUCHIAN, H. (1977): Val Thorens: perception et comportement dans une station de haute altitude, Institut de Géographie Alpine, Grenoble.

GUERIN, J.-P. et GUMUCHIAN, H. (1978): Les mythologies de la montagne, Revue de Géographie Alpine, p. 385-402. HOLZ, J.-M. (1980): Voyage dans l'espace, in Mélanges géographiques, A. BAILly et al., ed., Cahiers de géographie de Besançon, Les Belles Lettres, Paris.

MERENNE-SCHOUMAKER, B. (1974): La perception du centre-ville: le cas de Liège, Bulletin de la Société Géographique de Liège, no 10, p. 135-151.

MERENNE-SCHOUMAKER, B. (1979): Motivations des localisations commerciales au centre-ville: le cas des nouveaux commerces de l'hypercentre liègeois, Etudes et Expansion, p. 541-550.

METTON, A. et BERTRAND, M. (1974): Contribution à l'analyse géographique du quartier dans l'espace urbain, Revue de Géographie de Montréal, Vol. 28, no 1, p. 28-29.

METTON, A. (1977): Pratique commerciale facteur d'homogénéisation des espaces urbains, in Espace vécu et pratique commerciale, C. N. R. S., R. C. P., no 354.

MIOSSEC, J.-M. (1977): Image touristique comme introduction à la géographie du tourisme, Annales de Géographie, no 473, p. 55-70.

PAQUeTte, R. et al. (1974): Perception des changements dans l'environnement des résidents de la rue Portland, Bulletin de Recherche, no 18, Département de Géographie, Université de Sherbrooke.

PIOLLE, X. (1977): Espace vécu et stratégies commerciales dans le centre-ville, in Espace vécu et pratique commerciale, C. N. R. S., R. C. P., no 354, Caen.

PIVETEAU, J.-L. (1978): L'espace vécu chez le peuple hébreu, Geographica Helvetica, no 3, p. 141-144.

SÉMINAIRE DE RECHERCHE DE BENOUVILLE (1973): L'espace vécu, Supp. no 1 aux Cahiers du Département de l'Université de Caen. 